\title{
ONE POT SYNTHESIS OF PYRAZOLO PHTHALAZINE DIONE DERIVATIVES UNDER MICROWAVE IRRADIATION
}

\author{
S. R. Kolsepatil ${ }^{1}$, A. V. Sapkal ${ }^{2}$, S. Chandole ${ }^{3}$ and D. L. Lingampalle ${ }^{1, *}$ \\ ${ }^{1}$ Department of Chemistry, Vivekanand College, Aurangabad, (Maharashtra), India \\ ${ }^{2}$ Department of Chemistry, Vasantrao Naik College, Aurangabad, (Maharashtra), India \\ ${ }^{3}$ Department of Chemistry, S.G.B. College Purna (Jn.) Parbhani, (Maharashtra), India \\ *E-mail: lingampalle@ vivekanandcollege.edu.in
}

\begin{abstract}
In this method, we have reported the catalytic ability of boric acid as a green, eco-friendly catalyst for one-pot four component condensation reaction of phthalic anhydride, Monohydrate hydrazine, Malononitrile and substituted aromatic aldehyde was reported. The major synthetic protocol is the use of inexpensive, nontoxic, avoiding the use of harmful organic solvent, short reaction time, mild condition reaction, simple procedure, excellent yield and environmentally benign.

Keywords: Multicomponent Reaction, Green Synthesis, Pyrazolo Phthalazine Dione, Microwave Irradiation.

(C) RASĀYAN. All rights reserved
\end{abstract}

\section{INTRODUCTION}

Multicomponent condensation reactions (MCR) are more important in the synthesis of heterocyclic molecules. In presence of phthalazine and pyrazole heterocyclic ring system or fused ring with a different heterocyclic moiety, are in a number of pharmacologically emphasis molecules. In many cases, use of the classical method for the synthesis of heterocyclic compounds, however, these are simply no longer reaction time acceptable by current environmental and safety standard. All these reasons, the various possibilities offered by microwave synthesis are attractive high yield, fast or minimum time and environmentally benign are important advantages.

Nitrogen-containing bridgehead hydrazine heterocyclic compounds have attracted prominent interest due to their pharmacological properties, biological activities and clinical applications ${ }^{1,2,3,4,5,6}$. The pyrazolo phthalazine derivative expands in nature and their applications of biologically active pharmaceuticals, functional materials as well as agrochemicals. ${ }^{7-10}$ Pyrazolo phthalazine dione derivatives show various biological activities such as Anticonvulsant ${ }^{11}$, Cardiotonic ${ }^{12}$, vasorelaxant ${ }^{13}$, Cytotoxic $^{14}$, Anticancer ${ }^{15}$, Antifungal ${ }^{16}$, Anti-inflammatory ${ }^{17}$, Antiviral ${ }^{18}$, Antitumor ${ }^{19}$, Anticoagulant ${ }^{20}$, Antibacterial ${ }^{21}$ and Ant hypoglycemic activity ${ }^{22}$. To develop a simple method for synthesis of pyrazolo phthalazine dione is important in organic synthesis.

Earlier reports revealed that one-pot four component synthesis of pyrazolo phthalazine dione derivatives have been prepared by using different catalytic system such as $\mathrm{Et}_{3} \mathrm{~N}^{23}$, AL-KIT-6 ${ }^{23}$, [bmim] Br/PTSA ${ }^{24}$, $[\mathrm{bmim}] \mathrm{OH}^{25}, \quad \mathrm{NiCl}_{2} \cdot 6 \mathrm{H}_{2} \mathrm{O}^{26}, \quad \mathrm{InCl}_{3}{ }^{27}, \mathrm{CAN}^{28}, \quad\left[(\mathrm{DBU}) \mathrm{CH}_{3} \mathrm{COO}{ }^{29}, \mathrm{CuI} \mathrm{NPs}^{30}, \mathrm{Zn}(\mathrm{OAc})_{2}{ }^{31}\right.$ and $\mathrm{NiFe}_{2} \mathrm{O}_{4}{ }^{32}$. However, these strategies show different disadvantages related to green synthesis. The present method has less drawbacks such as short reaction time, use of a cheap catalyst and easy workup procedure.

Herein, we report the use of a catalyst as a boric acid non-toxic, inexpensive, easily available catalyst, for organic transformations to give excellent yield because of new numerous advantages associate with ecofriendly compound. The synthesis of pyrazolo phthalazine dione derivatives by one-pot four component condensation reaction of phthalic anhydride, Monohydrate hydrazine, substituted aromatic aldehyde and malononitrile (Scheme-1).

Rasayan J. Chem., 12(2), 415-420(2019)

http://dx.doi.org/10.31788/RJC.2019.1225035

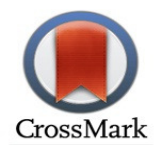




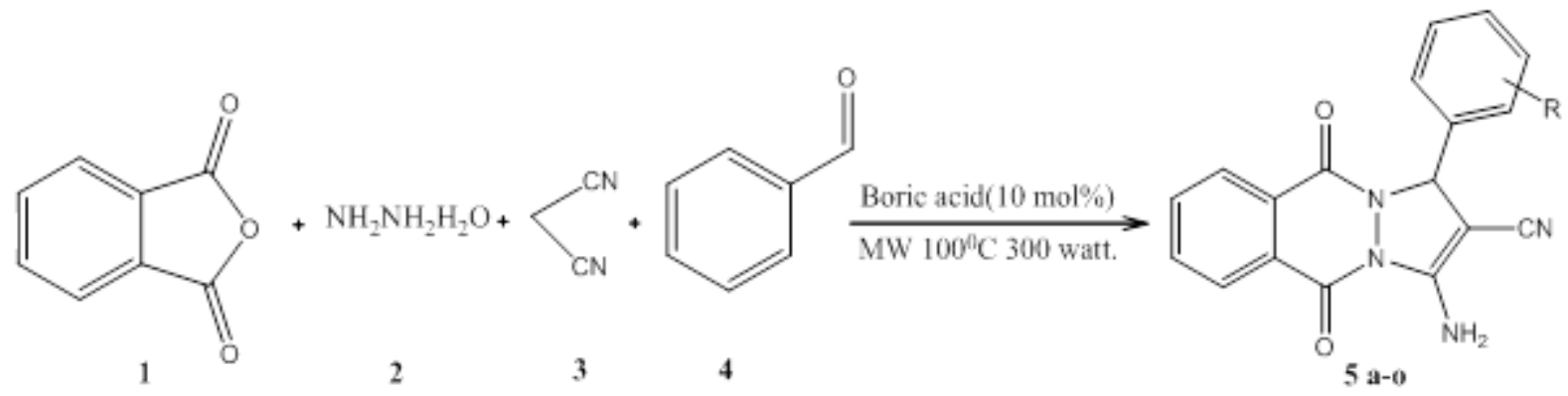

Scheme-1: Synthesis of Pyrazolo Phthalazine Dione Derivatives.

\section{EXPERIMENTAL}

\section{Materials and Methods}

All chemicals, solvents and reagents purchased from commercial sources.Melting points were taken in open capillary and are uncorrected. The reaction was carried out microwave synthesizer, mass-II, Sineo. ${ }^{1} \mathrm{H}$ NMR spectra were recorded on a Bucker 300 instrument and IR was recorded in $\mathrm{KBr}$ pellets on a Nicolet impact.

\section{General Reaction Procedure for Pyrazolo Phthalazine Dione Derivatives}

A mixture of phthalic anhydride $1(1 \mathrm{mmol})$ and monohydrate hydrazine $2(1 \mathrm{mmol})$ were mixed at $80^{\circ} \mathrm{C}$ for $15 \mathrm{~min}$. then added Malononitrile 3 (1 mmol), aromatic aldehyde 4 (1 mmol) and boric acid (10 mol\%) as a catalyst in round bottom flask were microwave irradiation at power 300 watt. At $100^{\circ} \mathrm{C}$ for $10 \mathrm{~min}$. after completion of the reaction, the reaction mixture was added ice cold water then it filtered and recrystallized from methanol.

\section{Spectral Data for Representative Compound}

3-Amino-1-(4-chlorophenyl)-5,10-dioxo-5,10-dihydro-1H-pyrazolo[1,2-b]phthalazine-2-carbonitrile (Yellow powder) (5b):MP 271-2740 $\mathrm{C}$, IR $\left(\mathrm{KBr}, \mathrm{cm}^{-1}\right) ; 3376,3260,2195,1660,1656 ;{ }^{1} \mathrm{H} \mathrm{NMR}\left(\mathrm{CDCl}_{3}\right.$, $300 \mathrm{MHz}) ; 6.15(1 \mathrm{H}, \mathrm{s}, \mathrm{CH}), 7.40-7.51(4 \mathrm{H}, \mathrm{m}, \mathrm{Ar}), 7.95-8.25\left(6 \mathrm{H}, \mathrm{m}, \mathrm{Ar} \& \mathrm{NH}_{2}\right),{ }^{13} \mathrm{CNMR}\left(\mathrm{CDCl}_{3}\right.$, 100MHz): $61.4,62.9,116.4,127.3,127.8,128.9,129.4,133.4,134.4,125.2,137.01,151.3,154.2,157.3$.

\section{3-Amino-1-(4-hydroxyphenyl)-5,10-dioxo-5,10-dihydro-1H-pyrazolo[1,2-b]phthalazine-2-} carbonitrile (Yellow powder)(5d):MP 270-273 ${ }^{\circ} \mathrm{C}$, IR (KBr, cm-1); 3373, 3260, 2198, 1664, 1569, ${ }_{1}^{1} \mathrm{H}$ NMR $\left(\mathrm{CDCl}_{3}, 300 \mathrm{MHz}\right) ; 6.03(1 \mathrm{H}, \mathrm{s}), 6.71-6.73(2 \mathrm{H}, \mathrm{d}, \mathrm{J}=8 \mathrm{~Hz}, \mathrm{Ar}), 7.23-7.25(2 \mathrm{H}, \mathrm{d}, \mathrm{J}=8 \mathrm{~Hz}, \mathrm{Ar}), 7.96-$ $8.25\left(6 \mathrm{H}, \mathrm{m}, \mathrm{Ar} \& \mathrm{NH}_{2}\right), 9.52(1 \mathrm{H}, \mathrm{s}),,{ }^{13} \mathrm{CNMR}\left(\mathrm{CDCl}_{3}, 100 \mathrm{MHz}\right): 61.5,62.7,115.01,116.3,126.7$, $127.4,128.4,128.7,128.9,133.5,134.6,150.6,153.6,156.5,157.7$.

\section{3-Amino-5,10-dioxo-1-p-tolyl-5,10-dihydro-1H-pyrazolo[1,2-b]phthalazine-2-carbonitrile(Yellow}

Powder)(5e): MP 252-254으, IR(KBr, cm-1) 3362,3260,2197,1657,1570, ${ }^{1} \mathrm{H}$ NMR $\left(\mathrm{CDCl}_{3}, 300 \mathrm{MHz}\right)$; 2.29(3H, S, CH3), 6.08(1H, S,CH), 7.15-7.34(4H, m, Ar), 7.95-8.26(6H, m, Ar \& NH $\mathrm{NH}_{2}$,

${ }^{13} \mathrm{C} \mathrm{NMR}\left(\mathrm{CDCl}_{3}, 100 \mathrm{MHz}\right) ; 121.3,62.4,64.01,123.2,123.5,127.5,127.7,127.9,128.2,128.8,130.3,130.9$, $133.4,134.7,135.01,136.6,153.01,154.8,157.01$

\section{RESULTS AND DISCUSSION}

The one-pot synthesis of pyrazolo phthalazine dione was possible by the one-pot four component cyclocondensation of phthalic anhydride (1), monohydrate hydrazine (2), aromatic aldehyde (3), and malononitrile (4) using boric acid as a catalyst. To optimized reaction condition using various solvents effects such as ethanol, tetrahydrofuran, dichloromethane, acetonitrile, methanol, ethylene glycol, PEG200, PEG-400, toluene, water and solvent-free condition. We examined the reaction proceed in ethanol and tetrahydrofuran the product was obtained with a lower yield which took it more time (Table-1, entry 1-2). Then other solvents were uses like as dichloromethane and acetonitrile, they gave the low yield and consumed more time. (Table-1, entry 3-4). The solvents are toluene and ethylene glycol take more time in 
complete reaction with less $48 \%$ and $67 \%$ yield respectively (Table-1, entry 5-6). The Solvents are PEG200 and PEG-400 give good yields but in increasing long time reaction (Table-1, entry 7-8). The water and methanol used in reaction as a solvent which takes more time and obtained low yield (Table 1, entry 9-10). Therefore, we decided the reaction goes to solvent-free condition and give excellent yield with lesser time.

Table-1:Synthesis of Compound 5a in the Presence of Different Solvents.

\begin{tabular}{c|c|c|c}
\hline Entry & Solvents & Time (Min.) & Yield $^{\mathrm{b}}(\%)$ \\
\hline 1 & $\mathrm{EtOH}$ & 45 & 60 \\
\hline 2 & $\mathrm{THF}$ & 120 & 40 \\
\hline 3 & $\mathrm{CH}_{2} \mathrm{Cl}_{2}$ & 50 & 53 \\
\hline 4 & $\mathrm{CH}_{3} \mathrm{CN}$ & 60 & 48 \\
\hline 5 & Toluene & 480 & 67 \\
\hline 6 & Ethylene Glycol & 300 & 87 \\
\hline 7 & PEG-200 & 80 & 89 \\
\hline 8 & $\mathrm{PEG}-400$ & 90 & 20 \\
\hline 9 & $\mathrm{H} 2 \mathrm{O}$ & 120 & 50 \\
\hline 10 & $\mathrm{MeOH}$ & 60 & 91 \\
\hline 11 & Solvent-free & 15 & .
\end{tabular}

aPhthalic anhydride $(1 \mathrm{mmol})$, hydrazine monohydrate $(1 \mathrm{mmol})$, benzaldehyde $(1 \mathrm{mmol})$ and malononitrile $(1 \mathrm{mmol})$. bIsolated yield.

To optimize all Lewis acid catalytic effect on multicomponent condensation reaction. The reaction was carried out in the same conditions, boric acid shows good catalytic role, which gave in product yield of 95\% (Table-1, entry14). When used $\mathrm{FeCl}_{2}$ and $\mathrm{MgO}$ as a catalyst, we observed5a in reasonably corresponding yield of $40 \%$ and $50 \%$ (Table-2, entry1-2). The reaction carried by $\mathrm{Et}_{3} \mathrm{~N}$ and $\mathrm{CuBr}$ they give the product in $45 \%$ and $61 \%$ yields (Table-2, entry $3-4$ ). The use of catalyst $\mathrm{CuCl}$ and $\mathrm{GaCl}_{3}$ take the same reaction time and product obtained 55\% and $35 \%$ yield respectively (Table-2, entry5-6). Another catalyst $\mathrm{ZnI}_{2}, \mathrm{MnCl}_{3}, \mathrm{Al}-\mathrm{KIT}-6, \mathrm{InCl}_{3}, \mathrm{CAN}$, [bmim] $\mathrm{Br} / \mathrm{PTSA}$ and these catalyst give low yield product and prolong reaction time $80 \%, 76 \%, 87 \%, 85 \%, 88 \%, 89 \%$, yields respectively (Table-2, entry 7-12). We determine the synthesis of pyrazolo phthalazine dione derivatives using boric acid using $10 \mathrm{~mol} \%$ give large amount of yields. (Table-2, entry 14). When $5 \mathrm{~mol} \%$ of boric acid was used to give $85 \%$ of yield. Hence, using $10 \mathrm{~mol} \%$ of boric acid was suitable with an excess amount of catalyst which shows and no improvement, no increase in the yield was observed.

Table-2: Comparison of Synthesis for Pyrazolo Phthalazine Dione Derivatives Using Boric Acid and Different

\begin{tabular}{c|c|c|c|c}
\multicolumn{7}{c}{ Catalyst. } \\
\hline Entry & Catalysts & Mol\% & Time(Min.) & Yield $^{\text {b } \%) ~}$ \\
\hline 1 & $\mathrm{FeCl}_{2}$ & 15 & 80 & 40 \\
\hline 2 & $\mathrm{MgO}$ & 10 & 80 & 50 \\
\hline 3 & $\mathrm{Et} N$ & 20 & 180 & 45 \\
\hline 4 & $\mathrm{CuBr}$ & 10 & 45 & 61 \\
\hline 5 & $\mathrm{CuCl}$ & 10 & 45 & 55 \\
\hline 6 & $\mathrm{GaCl}_{3}$ & 10 & 100 & 35 \\
\hline 7 & $\mathrm{ZnI}_{2}$ & 10 & 160 & 80 \\
\hline 8 & $\mathrm{MnCl}_{2}$ & 10 & 180 & 76 \\
\hline 9 & $\mathrm{Al}-\mathrm{KIT} 6$ & 10 & 240 & 87 \\
\hline 10 & $\mathrm{InCl}$ & 10 & 90 & 85 \\
\hline 11 & $\mathrm{CAN}$ & 5 & 80 & 88 \\
\hline 12 & {$[$ bmim]Br/PTSA } & 10 & 180 & 89 \\
\hline 13 & Boric acid & 5 & 20 & 85 \\
\hline 14 & Boric acid & 10 & 15 & 91 \\
\hline
\end{tabular}

aphthalic anhydride $(1 \mathrm{mmol})$, hydrazine monohydrate $(1 \mathrm{mmol})$, benzaldehyde $(1 \mathrm{mmol})$ and malononitrile $(1 \mathrm{mmol})$. bIsolated yield. 
RASĀYAN J. Chem.

Vol. 12 | No. 2 |415 - 420| April - June | 2019

To investigate the various substituted aromatic aldehyde containing an electron donating or electron withdrawing groups reacted with phthalic anhydride, hydrazine monohydrate and malononitrile using boric acid as a catalyst. Almost all reaction with different aromatic aldehyde and obtained product with desired high yield within lesser reaction time. Aromatic substituted aldehyde reacts faster and higher yield compared to aliphatic aldehyde (Table-3).

Table-3: Synthesis of Pyrazolo Phthalazine Dione Derivative ${ }^{a}$.

\begin{tabular}{|c|c|c|c|}
\hline Entry & Aldehydes & Time (min.) & Yield $^{\mathrm{b}}(\%)$ \\
\hline $5 a$ & & 10 & 91 \\
\hline $5 b$ & & 12 & 93 \\
\hline $5 c$ & & 13 & 90 \\
\hline $5 d$ & & 11 & 90 \\
\hline $5 e$ & & 15 & 91 \\
\hline $5 f$ & & 10 & 93 \\
\hline $5 \mathrm{~g}$ & & 11 & 94 \\
\hline $5 \mathrm{~h}$ & & 13 & 92 \\
\hline
\end{tabular}


RASĀYAN J. Chem.

Vol. 12 | No. 2 |415 - 420| April - June | 2019

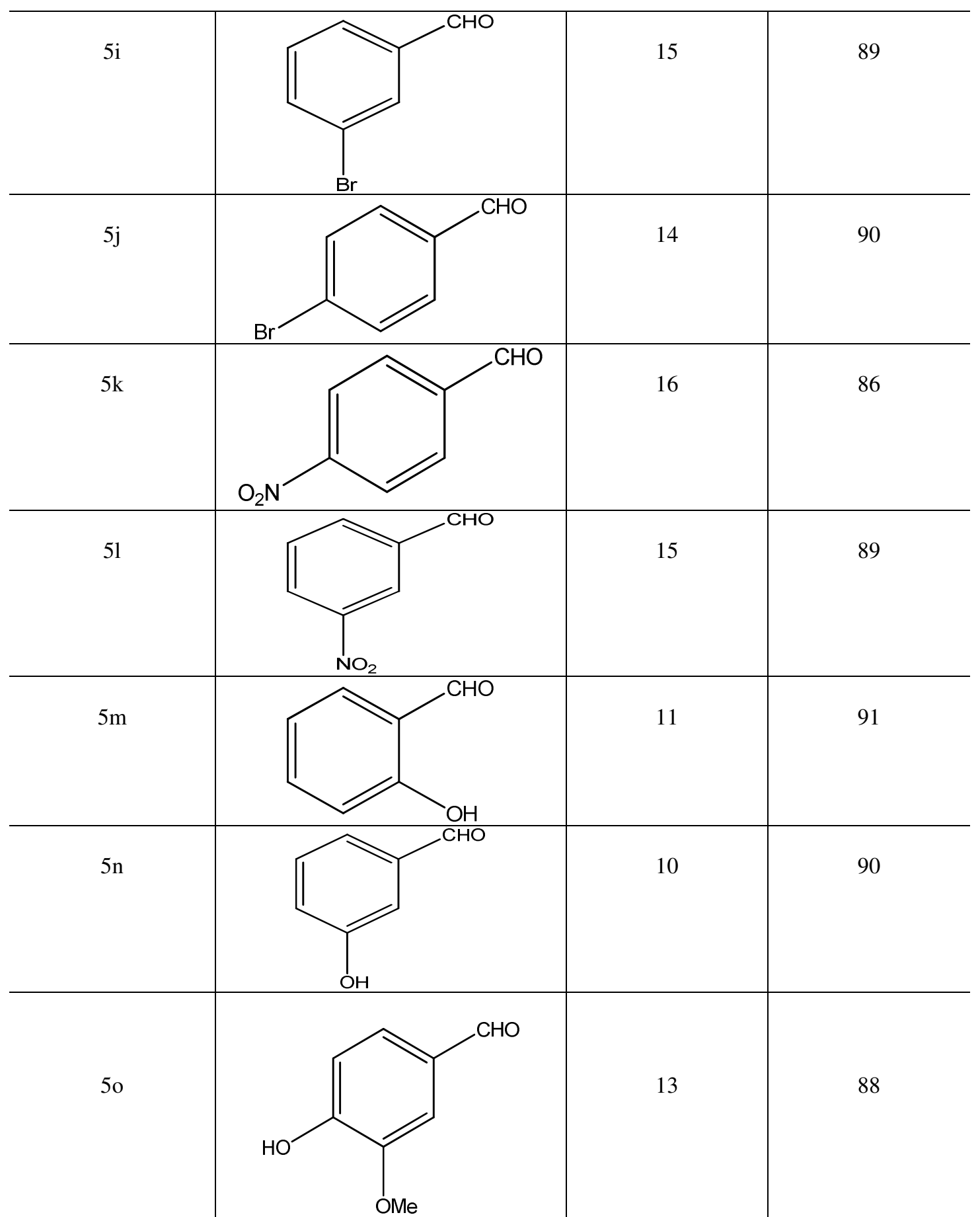

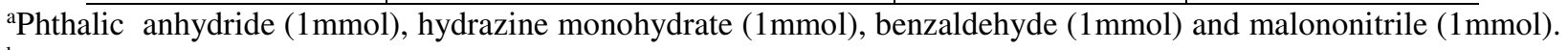
${ }^{\mathrm{b}}$ Isolated yield.

\section{CONCLUSION}

In summary, we have developed a one-pot four-component condensation reaction for novel pyrazolo phthalazine diones from substituted aromatic aldehyde with phthalahydrazide and malononitrile in presence boric acid under solvent-free conditions. The advantages of they are a simple procedure, inexpensive, easy workup, environmentally benign, excellent yield and shorter reaction time.

\section{ACKNOWLEDGMENT}

The authors thank the Principal, Vivekanand Arts, Sardar Dalipsingh Commerce and Science College, Aurangabad, for permitting the use of college research infrastructure. 
RASĀYAN J. Chem.

Vol. 12 | No. 2 |415 - 420| April - June | 2019

\section{REFERENCES}

1. W. R. Vaughan, Chem. Rev., 43, 447 (1948), DOI: 10.1021/cr60136a003

2. R.A. Clement, J. Org. Chem.,25, 1724 (1960)

3. H.W. Heine, R. Henrie, L. Heitz, S.R. Kovvali, J. Org. Chem.,39, 3187 (1974)

4. H.W. Heine, L.M. Aclawski, S.M. Bonser, G.D. Wachob, J. Org. Chem.,41, 3229(1976), DOI: 10.1021/jo008829002

5. T. Sheradsky, R. Moshenberg, J. Org. Chem.,51, 3123(1986), DOI: 10.1021/jo00366a008

6. L.N. Jungheim, S.K. Sigmund, J. Org. Chem.,52, 4007 (1987)

7. E.C. Franklin, Chem. Rev.,16, 305(1935), DOI:10.1021/cr600559001

8. F.W. Bergstrom, Chem. Rev.,35, 77(1944), DOI:10.1021/cr601119001

9. F.W. Lichtenthaler, Acc. Chem. Res.,35, 728(2002), DOI:10.1021/ar010071i

10. M.R. Nabid, S.J.Tabatabaie, R. Gahremanzadeh, A. Bazgir, Ultrason. Sonochem.,17, 159(2010), DOI: 10.1016/j.ultsonch.2009.06.012

11. S. Grasso, G. DeSarro, N. Micale, M. Zappala, G. Puja, M. Baraldi, C.Demicheli, J. Med. Chem.,43, 2851(2000), DOI:10.1021/jm001002x

12. Y. Nomoto, H. Obase, H. Takai, M. Teranishi, J. Nakamura, K. Kubo, Chem. Pharma. Bull.,38, 2179(1990), DOI: $10.1248 / \mathrm{cpb} .38 .2179$

13. N. Watanabe, Y. Kabasawa, Y. Takase, M. Matsukura, K. Miyazaki, H. Ishihara, K. Kodama, H. Adachi, J. Med. Chem.,41, 3367(1998), DOI:10.1021/jm970815r

14. J.S. Kim, H.K. Rhee, H.J. Park, S.K. Lee, C. Lee, H.P. Choo, Bioorg. Med. Chem.,16, 4545(2008), DOI: 10.1016/j.bmc.2008.02.049

15. S.S. El-Saka, A.H. Soliman, A.M. Imam, Afinidad, 66, 167(2009)

16. C. Ryu, R. Park, M. Ma, J. Nho, Bioorg. Med.Chem. Lett., 17, 2577(2007), DOI: 10.1016/j.bmcl.2007.02.003

17. T. Nakamura, M. Sato, H. Kakinuma, J. Med. Chem., 46, 5416(2003), DOI: 10.1021/jm020557k

18. M.J. Genin, C. Biles, B.J. Keiser, J. Med. Chem., 43, 1034(2000), DOI:10.1021/jm990383f

19. F.Wei, B.X. Zhao, B. Huang, Bioorg. Med. Chem. Lett., 16, 6342(2006), DOI: 10.1016/j.bmcl.2006.09.008

20. Y. Xia, Z.W. Dong, B.X. Zhao, Bioorg. Med. Chem., 15, 6893(2007), DOI: 10.1016/j.bmc.2007.08.021

21. P.C. Lu, J. Sun, Y. Yang, H.L. Zhu, Bioorg. Med. Chem. Lett., 15, 4657(2010), DOI: 10.1016/j.bmcl.2010.05.105

22. N. Cho, M. Kamaura, T. Yogo, H. Imoto, WO 2009139340

23. G. Karthikeyana, A. Pandurangan, J. Mol. Catal. A: Chem., 361-362, 58(2012), DOI: 10.1016/j.molcata.2012.05.003

24. R. Ghahremanzadeh, G. Imani Shakibaei, A. Bazgir, Synlett., 8, 1129(2008), DOI: 10.1021/cc900130a

25. D.S. Raghuvanshi, K.N. Singh, Tetrahedron Lett., 52, 5702(2011), DOI: 10.1039/c7ra02350b

26. S. Song, J. Zhong, Y. He, Z. Guan, Tetrahedron Lett., 53, 7075(2012), DOI: 10.1016/j.tetlet.2012.10.063

27. Y. D. Reddy, B. Suryanarayana, Ch. V.R. Reddy, P.K. Dubey, Heterocyclic Lett., 4(3)341(2014)

28. M. Kidwai, R. Chauhan, J. Heterocyclic Chem., 51, 1689(2014), DOI: 10.1002/jhel.1809

29. H.R. Shaterian, M. Mohammadnia, J. Mol. Liq., 173, 55(2012), DOI: 10.1016/j.molliq.2012.06.007

30. J. Safaei-Ghomi, H. Shahbazi-Alavi, A. Ziarati, R. Teymuri, M.R. Saberi, Chin. Chem. Lett., 25, 401(2014) , DOI:10.1016/j.cclet.2013.11.046

31. F. Mohamadpour, M. Lashkari, R. Heydari, N. Hazeri. Indian Journal of Chemistry, 57-B 843(2017)

32. V.V. Dabholkar, S.K. Kurade, K.S. Badhe, Der PharmaChemica., 10(6), 135(2018)

[RJC-5035/2018] 